\title{
Observational Retrospective Study of Altered Biodistribution of Tositumomab and ${ }^{131}$ I-Tositumomab
}

\author{
Richard L. Wahl ${ }^{1}$, Thierry J. Horner ${ }^{2}$, Thomas S. Lin² ${ }^{2}$ and Mark S. Kaminski ${ }^{3}$ \\ ${ }^{1}$ Department of Medical Oncology, Johns Hopkins University, Baltimore, Maryland; ${ }^{2}$ Oncology Research and Development, \\ GlaxoSmithKline, Collegeville, Pennsylvania; and ${ }^{3}$ Department of Internal Medicine, University of Michigan, Ann Arbor, Michigan
}

The tositumomab/131/-tositumomab radioimmunotherapy regimen is
administered as a dosimetric dose followed by a therapeutic dose.
The biodistribution of the dosimetric dose is assessed by quantitative
calculations of whole-body residence time (TBRT) and visual exami-
nation of whole-body $\mathrm{Y}$-camera images, to determine the adminis-
tered radioactivity dose and whether a therapeutic dose can be
administered. We investigated whether altered biodistribution of
131 I-tositumomab could be identified using quantitative TBRT.
Methods: BioClinica, Inc., provided -camera images to an indepen-
dent reviewer to assess altered ${ }^{131}$ I-tositumomab biodistribution in
patients reported to a registry. Results: Of 2,649 therapeutic doses,
5 (0.2\%) were cancelled because of altered biodistribution as deter-
mined by Y-camera images and TBRT. Of these, 3 Y-camera images
were assessed by the independent reviewer; one showed altered bio-
distribution ( $0.04 \%)$ and was in agreement with the TBRT on-site cal-
culation. Conclusion: TBRT alone should be used to determine altered
biodistribution and hence whether to administer the therapeutic dose.
Key Words: tositumomab; altered biodistribution; therapeutic dose

J Nucl Med 2015; 56:1800-1803

DOI: 10.2967/jnumed.115.156190

\section{$\mathbf{T}$} ositumomab/ ${ }^{131}$ I-tositumomab (TST/131 I-TST $\quad$ [Bexxar]; GlaxoSmithKline) is an anti-CD20 antibody regimen and was approved in the United States in 2003 for the treatment of patients with rituximab-refractory, CD20-positive, low-grade non-Hodgkin lymphoma with or without transformation (1-4). The TST $/{ }^{131}$ I-TST regimen consists of a 2-part dosimetric step, followed 7-14 d later by a 2-part therapeutic step (1). Whole-body clearance is patientspecific, and high doses of ${ }^{131}$ I-TST are known to cause myelosuppression, nausea, infections, and cardiotoxicity (5). Therefore, ${ }^{131} \mathrm{I}-\mathrm{TST}$ biodistribution is assessed after the dosimetric dose to determine the therapeutic dose to be given; if the biodistribution of the dosimetric dose is abnormal, the therapeutic dose is not administered. Normal biodistribution occurs in the heart and major blood vessels and, to a lesser extent, in the liver and spleen, with possible uptake in the thyroid, kidney, and urinary bladder (1).

Biodistribution of TST/ ${ }^{131} \mathrm{I}$-TST after the dosimetric dose is assessed both by quantitative determination of whole-body resi-

Received Feb. 18, 2015; revision accepted Aug. 17, 2015.

For correspondence or reprints contact: Thierry J. Horner, Oncology Research and Development, GlaxoSmithKline, 1250 S. Collegeville Rd., Collegeville, PA 19426-0989.

E-mail: thierry.j.horner@gsk.com

Published online Sep. 3, 2015.

COPYRIGHT (c) 2015 by the Society of Nuclear Medicine and Molecular Imaging, Inc. dence time (TBRT) and by visual examination of whole-body $\gamma$-camera images at 3 time points. However, the necessity of acquiring more than one $\gamma$-camera image to assess biodistribution before administration of radioimmunotherapy has been questioned by data from the Zevalin Imaging Registry (6). These data demonstrated that a single $\gamma$-camera image obtained within $24 \mathrm{~h}$ of administration of the test dose of ${ }^{111}$ In-ibritumomab tiuxetan successfully identified all cases of altered biodistribution (6).

We performed an observational, retrospective study to determine the frequency of patients with an altered biodistribution of ${ }^{131} \mathrm{I}$-TST after receiving the dosimetric dose of TST/ ${ }^{131} \mathrm{I}$-TST in the postmarketing setting. In addition, we wanted to evaluate the clinical benefit of visually assessing $\gamma$-camera images obtained after the dosimetric dose to determine the biodistribution of ${ }^{131} \mathrm{I}-$ TST and hence whether to administer the therapeutic dose.

\section{MATERIALS AND METHODS}

\section{Study Design}

This was an observational, retrospective study (GlaxoSmithKline study BEX114606) with anonymized data from patients who received the dosimetric dose of TST/ ${ }^{131}$ I-TST in the commercial setting between June 2003 and February 2010. Commercial orders for TST/ ${ }^{131}$ I-TST were retrospectively assessed to determine the total number of patients who received the dosimetric dose, the reasons for cancellation of the therapeutic dose, and the frequency of patients with a reported altered biodistribution. The frequency of cancellations was summarized according to medical reasons or other reasons. Whole-body $\gamma$-camera images and dosimetry calculations of the TBRT of patients who did not receive the therapeutic dose because of altered biodistribution were evaluated by an independent nuclear medicine physician. Images from patients with an expected biodistribution were not independently reviewed.

TST $/{ }^{131} \mathrm{I}$-TST was administered according to its prescribing information (1). The dosimetric dose consisted of $450 \mathrm{mg}$ of unlabeled TST (in $50 \mathrm{~mL}$ of $0.9 \% \mathrm{NaCl}$ solution) administered intravenously over $1 \mathrm{~h}$, followed immediately by $35 \mathrm{mg}$ of TST labeled with $185 \pm$ $18.5 \mathrm{MBq}(5 \pm 0.5 \mathrm{mCi})$ of ${ }^{131} \mathrm{I}$ (in $30 \mathrm{~mL}$ of $0.9 \% \mathrm{NaCl}$ solution) administered intravenously over $20 \mathrm{~min}$. The therapeutic dose consisted of the same unlabeled TST dose, followed by $35 \mathrm{mg}$ of TST labeled with a patient-specific activity of ${ }^{131} \mathrm{I}$ (in $30 \mathrm{~mL}$ of $0.9 \% \mathrm{NaCl}$ solution). The required activity of ${ }^{131} \mathrm{I}$ to deliver the desired whole-body dose of radiation was calculated according to the patient platelet count: $65 \mathrm{cGy}$ if the platelet count was $100,000-149,999 / \mathrm{mm}^{3} ; 75 \mathrm{cGy}$ if the platelet count was at least $150,000 / \mathrm{mm}^{3}(1)$.

\section{Assessment of Dosimetry and Biodistribution \\ After the dosimetric dose, whole-body $\gamma$-camera counts and whole- body $\gamma$-camera images were obtained at 3 time points: within $1 \mathrm{~h}$ of infusion and before urination, 2-4 d after infusion of the dosimetric dose, and 6-7 d after infusion of the dosimetric dose. TBRT was the}


time in hours at which the activity was $37 \%$ of that at time zero; the activity was measured by whole-body $\gamma$-camera counts.

\section{Data Collection and Management}

Records of completed and cancelled TST $/{ }^{131}$ I-TST commercial orders were maintained by the GlaxoSmithKline Speciality Service Centre using the Bexxar Operations and Order Tracking System. Reasons for cancellation of the therapeutic dose were prospectively recorded by the GlaxoSmithKline Speciality Service Centre at the time of cancellation and retrospectively confirmed by the treatment site investigator. GlaxoSmithKline used anonymized dosimetry calculations and $\gamma$-camera images from the medical centers to assess the dosimetry and biodistribution for reported cases of altered ${ }^{131}$ I-TST biodistribution.

BioClinica, Inc., managed the image files and coordinated the independent review of biodistribution.

\section{Independent Review of y-Camera Images and Time Calculations}

Whole-body $\gamma$-camera images were independently reviewed by a nuclear medicine physician who did not know the results of the original biodistribution assessment performed at the treatment sites and did not receive patient dosimetry data. The independent reviewer prepared a description of the ${ }^{131}$ I-TST biodistribution at each of the 3 time points examined for each subject and, on the basis of criteria in the prescribing information (Table 1) (1), determined whether the ${ }^{131} \mathrm{I}-$ TST biodistribution was expected or altered.

The TBRT of ${ }^{131}$ I-TST after administration of the dosimetric dose was validated by an independent reviewer in patients deemed to have an altered biodistribution. An altered biodistribution was defined as a TBRT of less than $50 \mathrm{~h}$ or more than $150 \mathrm{~h}(1)$.

\section{RESULTS}

\section{Treatment Information}

In total, 2,649 patients received a dosimetric dose of TST/ ${ }^{131} \mathrm{I}$-TST in the postmarketing setting between June 2003 and February
2010, of whom 2,541 (96\%) also received a therapeutic dose. Of the remaining 108 patients, $105(4 \%)$ had their therapeutic dose cancelled and $3(0.1 \%)$ were rescheduled. One patient rescheduled the therapeutic dose because of a medical condition (shingles). Medical reasons for cancellation included patient illness $(n=$ $47,1.8 \%)$, adverse event during or after the dosimetric dose $(n=$ $12,0.5 \%)$, low platelet count $(n=10,0.4 \%)$, unrelated medical conditions $(n=8,0.3 \%)$, altered biodistribution $(n=5,0.2 \%)$, and death $(n=2,0.1 \%)$. One death was caused by disease progression and the other by an anaphylactic reaction. Nonmedical reasons for cancellation were logistics $(n=11,0.4 \%)$, patient compliance with radiation safety $(n=5,0.2 \%)$, the physician's decision $(n=4,0.2 \%)$, and the patient's decision $(n=1,0.04 \%)$.

\section{Altered Biodistribution Reported by the Treatment Sites}

In total, 5 patients $(0.2 \%)$ who received the dosimetric dose of TST $/{ }^{131}$ I-TST were determined to have altered biodistribution by the treatment site investigator because of reported lung uptake $(n=$ $3)$, reported stomach uptake $(n=1)$, and a TBRT of $45 \mathrm{~h}(n=1)$.

\section{Independent Review of y-Camera Images and Time Calculations}

Whole-body $\gamma$-camera images were available for 3 of the 5 patients who had altered biodistribution after the dosimetric dose according to the site investigator. Descriptions of the visual assessment of $\gamma$-camera images are presented in Table 2. The independent reviewer concluded that 1 of the 3 patients had an altered biodistribution, representing $0.04 \%$ of all patients receiving the dosimetric dose.

Dosimetry calculations were performed by the treatment site investigator for 3 of the 5 patients deemed to have an altered biodistribution. The TBRTs were calculated as 45,101 , and $154 \mathrm{~h}$ (Table 2). These dosimetry calculations were independently assessed, and all 3 were found to be accurate. Hence, 2 patients

TABLE 1

Expected and Altered Biodistribution of ${ }^{131}$ I-TST (1)

\section{Biodistribution}

Expected

\begin{tabular}{l}
\hline Altered \\
\hline \\
\hline \\
\hline${ }^{*}$ Image taken on day 0. \\
\hline IImage taken on day 2,3, or 4. \\
${ }^{ \pm}$Image taken on day 6 or 7.
\end{tabular}

Most activity in blood pool on first* image; with uptake in normal liver and spleen less than that in heart on day of dosimetric dose

Significantly decreased activity in blood pool during second ${ }^{\dagger}$ and third ${ }^{\ddagger}$ images Decreased accumulation in normal liver and spleen during second ${ }^{\dagger}$ and third ${ }^{\ddagger}$ images

Possible uptake in thyroid, kidney, and urinary bladder, with minimal uptake in lungs during second ${ }^{*}$ and third ${ }^{\ddagger}$ images

Possible increased intensity at known lymphoma sites during second ${ }^{\star}$ and third ${ }^{\dagger}$ images No activity visualized in blood pool on first ${ }^{\ddagger}$ image

Diffuse, intense tracer uptake in liver or spleen or uptake suggestive of urinary obstruction on first ${ }^{\ddagger}$ image

Diffuse uptake in normal lung greater than that in blood pool on first* image

Uptake suggestive of urinary obstruction

Diffuse uptake in normal lung greater than that in blood pool

TBRT $<50 \mathrm{~h}$ or $>150 \mathrm{~h}$ 
TABLE 2

Results of Patients Not Receiving Therapeutic Dose Because of Altered Biodistribution

\begin{tabular}{|c|c|c|c|c|}
\hline \multirow[b]{3}{*}{ Patient no. } & \multicolumn{2}{|c|}{ Assessment by treatment site } & \multirow{2}{*}{\multicolumn{2}{|c|}{ Assessment by independent review }} \\
\hline & \multirow{2}{*}{$\begin{array}{l}\text { Biodistribution } \\
\text { by visual } \\
\text { inspection of y } \\
\text { images }\end{array}$} & \multirow{2}{*}{$\begin{array}{l}\text { TBRT } \\
\text { (h) }\end{array}$} & & \\
\hline & & & Visual assessment of $y$-camera images & Biodistribution result \\
\hline \multirow[t]{3}{*}{1} & Unknown* & 45 & $\begin{array}{l}\text { Day 0: Most activity in blood pool, and uptake in } \\
\text { normal liver and spleen less than in heart }\end{array}$ & Altered \\
\hline & & & $\begin{array}{l}\text { Day 4: Blood pool not visualized; diffuse, increased } \\
\text { uptake in liver and spleen }\end{array}$ & \\
\hline & & & $\begin{array}{l}\text { Day 7: Altered biodistribution best appreciated by } \\
\text { comparison of } 3 \text { image sets }\end{array}$ & \\
\hline \multirow[t]{3}{*}{2} & $\begin{array}{l}\text { Altered by lung } \\
\text { uptake }\end{array}$ & 154 & $\begin{array}{l}\text { Day 0: Most activity in blood pool; uptake in normal } \\
\text { liver and spleen less than in heart }\end{array}$ & Expected \\
\hline & & & $\begin{array}{l}\text { Day 2: Most activity in blood pool; uptake in normal } \\
\text { liver and spleen less than in heart }\end{array}$ & \\
\hline & & & $\begin{array}{l}\text { Day 6: Most activity in blood pool; uptake in normal } \\
\text { liver and spleen less than in heart; normal } \\
\text { biodistribution best visualized on day } 0\end{array}$ & \\
\hline \multirow[t]{3}{*}{3} & $\begin{array}{l}\text { Altered by lung } \\
\text { uptake }\end{array}$ & 101 & $\begin{array}{l}\text { Day 0: Most activity in blood pool; uptake in normal } \\
\text { liver and spleen less than in heart }\end{array}$ & Expected \\
\hline & & & $\begin{array}{l}\text { Day 3: Most activity in blood pool; uptake in normal } \\
\text { liver and spleen less than in heart; tumor } \\
\text { targeting }\end{array}$ & \\
\hline & & & $\begin{array}{l}\text { Day 6: Most activity in blood pool; uptake in normal } \\
\text { liver and spleen less than in heart; tumor } \\
\text { targeting; normal biodistribution best visualized } \\
\text { on day } 3\end{array}$ & \\
\hline 4 & $\begin{array}{l}\text { Altered by } \\
\text { stomach } \\
\text { uptake }\end{array}$ & NR & NA & - \\
\hline 5 & $\begin{array}{l}\text { Altered by lung } \\
\text { uptake }\end{array}$ & NR & NA & - \\
\hline
\end{tabular}

*Deemed to have altered biodistribution because of TBRT of $45 \mathrm{~h}$.

$\mathrm{NR}=$ not reported; NA $=$ not available.

( $0.08 \%$ of total) had a TBRT outside $50-150$ h (i.e., defined as altered biodistribution).

\section{DISCUSSION}

This study suggests that patients rarely have an altered biodistribution of ${ }^{131} \mathrm{I}$-TST when treated with $\mathrm{TST} /{ }^{131} \mathrm{I}$-TST in the postmarketing setting $(\leq 0.2 \%)$.

The investigators' and reviewers' assessments of altered biodistribution based on whole-body $\gamma$-camera images were conflicting; only 1 of 3 patients who were identified by investigators to have altered biodistribution by whole-body $\gamma$-camera images was confirmed to have altered biodistribution on independent review. Thus, assessments of abnormal biodistribution by whole-body $\gamma$-camera images were not reproducible. The increased lung uptake of ${ }^{131}$ I-TST on early images was not verified by central review, suggesting that this criterion for altered biodistribution is not particularly reliable.

Conversely, TBRT calculated by the site investigators was confirmed by the independent reviewer and hence deemed reproducible.
Furthermore, TBRT is substantially based on the same factors as blood clearance and so provides an objective, indirect method to determine biodistribution.

We believe these results demonstrate that visual assessment of images provides minimal clinical benefit compared with TBRT. Furthermore, relying on $\gamma$-camera images may bring about inaccurate visual assessments of biodistribution resulting in therapy being withheld from patients, especially if the only finding is increased tracer uptake in the lungs. Because deiodination of antibodies can occur, radiotracer accumulation in the stomach may, in some instances, be an acceptable biodistribution as well. Hence, in one case, this "altered biodistribution" might more appropriately be considered within the reference range of biodistribution values.

Admittedly, data for 2 patients are missing. However, even with a worst-case assumption that these 2 patients had an altered biodistribution, less than $0.1 \%$ of patients studied by the imaging dosimetry method would have had an altered biodistribution detectable only by $\gamma$-camera imaging. 


\section{CONCLUSION}

These data indicate that true altered biodistribution of TST/ ${ }^{131}$ I-TST is very rare and suggest that nonimaging methods, such as measuring TBRT with a probe system or abbreviated whole-body imaging approaches, may be sufficient to identify the rare occurrences of altered biodistribution and allow individualized radiation dose delivery of TST/ ${ }^{131}$ I-TST. Such a simplification in dosimetry assessment could make radiopharmaceutical treatment administration simpler and less expensive than recording sequential $\gamma$-camera images.

\section{DISCLOSURE}

The costs of publication of this article were defrayed in part by the payment of page charges. Therefore, and solely to indicate this fact, this article is hereby marked "advertisement" in accordance with 18 USC section 1734. The study was funded by GlaxoSmithKline. Thierry J. Horner is an employee of GlaxoSmithKline and owns stock/stock options in the company. Thomas S. Lin was an employee of GlaxoSmithKline and owned stock/stock options in the company at the time of the study. No other potential conflict of interest relevant to this article was reported.

\section{ACKNOWLEDGMENTS}

Medical writing assistance was provided by Joanne Ashworth (Fishawack Indicia Ltd.), funded by GlaxoSmithKline.

\section{REFERENCES}

1. Bexxar ${ }^{\circledR}$ (tositumomab and iodine I-131 tositumomab) [prescribing information]. GlaxoSmithKline website. http:/www.accessdata.fda.gov/scripts/cder/drugsatfda/ index.cfm?fuseaction=Search.DrugDetails. Approved June 27, 2003. Accessed September 2, 2015.

2. Illidge T, Morschhauser F. Radioimmunotherapy in follicular lymphoma. Best Pract Res Clin Haematol. 2011;24:279-293.

3. Kaminski MS, Tuck M, Estes J, et al. ${ }^{131}$ I-tositumomab therapy as initial treatment for follicular lymphoma. $N$ Engl J Med. 2005;352:441-449.

4. Kaminski MS, Zelenetz AD, Press OW, et al. Pivotal study of iodine I 131 tositumomab for chemotherapy-refractory low-grade or transformed low-grade B-cell non-Hodgkin's lymphomas. J Clin Oncol. 2001;19: 3918-3928.

5. Press OW, Eary JF, Appelbaum FR, et al. Radiolabeled-antibody therapy of B-cell lymphoma with autologous bone marrow support. N Engl J Med. 1993;329:12191224.

6. Conti PS, White C, Pieslor P, et al. The role of imaging with ${ }^{111} \mathrm{In}$-ibritumomab tiuxetan in the ibritumomab tiuxetan (Zevalin) regimen: results from a Zevalin imaging registry. J Nucl Med. 2005;46:1812-1818. 